\title{
Clinical Study Slowing of Motor Imagery after a Right Hemispheric Stroke
}

\author{
Francine Malouin, ${ }^{1}$ Carol L. Richards, ${ }^{1}$ and Anne Durand ${ }^{2}$ \\ ${ }^{1}$ Department of Rehabilitation, Laval University and Center for Interdisciplinary Research in Rehabilitation and \\ Social Integration (CIRRIS), IRDPQ 525 Boulevard Hamel East, Quebec City, QC, Canada G1M 2 S8 \\ ${ }^{2}$ Institut de Réadaptation en Déficience Physique de Québec, 525 Boulevard Hamel East, Quebec City, QC, Canada G1M 2 S8
}

Correspondence should be addressed to Francine Malouin, francine.malouin@rea.ulaval.ca

Received 13 October 2011; Revised 11 January 2012; Accepted 7 February 2012

Academic Editor: Keh-chung Lin

Copyright ( $\odot 2012$ Francine Malouin et al. This is an open access article distributed under the Creative Commons Attribution License, which permits unrestricted use, distribution, and reproduction in any medium, provided the original work is properly cited.

The temporal congruence between real and imagined movements is not always preserved after stroke. We investigated the dependence of temporal incongruence on the side of the hemispheric lesion and its link with working memory deficits. Thirtyseven persons with a chronic stroke after a right or left hemispheric lesion (RHL: $n=19$; LHL: $n=18$ ) and 32 age-matched healthy persons (CTL) were administered a motor imagery questionnaire, mental chronometry and working memory tests. In contrast to persons in the CTL group and LHL subgroup, persons with a RHL had longer movement times during the imagination than the physical execution of stepping movements on both sides, indicating a reduced ability to predict movement duration (temporal incongruence). While motor imagery vividness was good in both subgroups, the RHL group had greater visuospatial working memory deficits. The bilateral slowing of stepping movements in the RHL group indicates that temporal congruence during motor imagery is impaired after a right hemispheric stroke and is also associated with greater visuospatial working memory deficits. Findings emphasize the need to use mental chronometry to control for movement representation during motor imagery training and may indicate that mental practice through motor imagery will have limitations in patients with a right hemispheric stroke.

\section{Introduction}

Motor imagery, which is the imagining of actions without their execution, can be defined as an active process during which the representation of a specific action is internally reproduced within working memory without any overt output [1]. The rationale for using motor imagery in the rehabilitation of motor impairments likely arises from the functional correlates that motor imagery shares with the execution of physical movement. For instance, the duration of imagined movements correlates with the duration of real movements $[2,3]$, simulation of movements evokes similar autonomic responses $[4,5]$, and, more importantly, the imagination of an action engages largely similar neural networks as its physical execution, notably motor and premotor areas and parietal cortices [6-8]. These observations suggest that real and covert movements during motor imagery obey similar principles and share similar mechanisms.
Temporal characteristics of motor imagery have been extensively studied with mental chronometry in healthy individuals [9]. Studies that examined the temporal relationship between the physical execution of a motor task (i.e., writing, walking, pointing, stepping) and the imagination of the same task (mental chronometry) have found that the imagination and execution times are generally similar (temporal congruence or functional equivalence). In addition, it has been shown that Fitt's law, which states that more difficult movements take more time to produce physically than easier ones, applies also to imagined movements [3] indicating that the timing of movements, either performed physically or imagined, is subject to common laws and principles [10]. For this reason, mental chronometry has been used by many to examine the effects of brain lesions on the temporal organization of motor imagery [11-16], to assess MI ability $[9,17,18]$ and to control whether patients are engaged in mental rehearsal during mental practice [19]. 
While some studies have shown that, following a unilateral lesion of the motor cortex, movements are slower on the affected limbs during both the physical execution and mental simulation of the same movement confirming temporal congruence $[11,12]$, others have reported that patients with lesions restricted to the superior regions of the parietal cortex could not predict through mental imagery the time necessary to perform various finger movements and visually guided pointing gestures, suggesting that the parietal cortex is important for the ability to generate mental movement representations [13]. More recently, temporal incongruence has been described following stroke during motor imagery of upper limb [14-16] and lower limb [14] movements. In these studies, in contrast to control subjects, patients showed simulation times that were longer than real movement times (overestimation of movement duration) indicative of a slowing of the motor imagery process, especially in persons with right hemispheric $(\mathrm{RH})$ strokes $[15,16]$. In the six patients with RH stroke showing a slowing of motor imagery, the corticomotor excitability, as measured with transcranial magnetic stimulation, was not increased during imagery conditions [16] suggesting that facilitation of motor cortex excitability during motor imagery depends on input from regions of the right hemisphere which can be disrupted by right hemispheric stroke.

Because in prior studies limb dominance of the patients was not controlled [14-16] and the number of patients with a right and a left hemispheric lesion was small $[15,16]$ or uneven [14], no definite conclusion can be drawn as to the impact of the lesion side on temporal incongruence between real and imagined movements. The present study sought to extend these prior findings in a larger sample of patients with right and left hemispheric stroke having similar limb dominance. In addition, since motor imagery is an active process during which the representation of a specific action is internally reproduced within working memory [1] and given the role of the right cerebral hemisphere in image generation [20] and visuospatial working memory [21, 22], we also examined whether lesion side had an impact on visuospatial working memory performance. It was hypothesized that right hemispheric lesions (RHLs) would be associated with both a slowing of motor imagery and greater visuospatial working memory deficits. The aims of this study were to determine whether the slowing of motor imagery after stroke is linked to the side of the cerebral lesion and, further, if it is associated with an impairment of visuospatial short-term memory.

\section{Methods}

2.1. Participants and Design. The study included a group of 37 persons who sustained a cortical or a subcortical lesion (CVA group) of the right $(n=19)$ or the left $(n=$ 18) hemisphere and a group of 32 age-matched healthy individuals (CTL group). To be included in the CVA group, subjects had to present a hemiparesis consecutive to a stroke and be right handed. The patients with the following conditions were excluded: (1) lesions in the cerebellum or midbrain (MRI or CT scan); (2) severe aphasia, based on the clinical evaluation of the speech therapist; (3) severe perceptual problems (i.e., hemineglect) discerned by clinical tests performed by the treating occupational therapist and severe cognitive impairments determined by the neuropsychologist evaluation; (4) other neurological conditions (i.e., Parkinson's disease, dementia). To be included in the CTL group subjects had to be between 35 and 80 years old, without physical or cognitive impairments. All subjects gave informed, written consent for their participation in the study and the protocol was approved by the Ethics Committee of the Rehabilitation Institute where the study took place.

2.2. Assessment Procedures. Prior to the administration of the chronometric tests, the hand and leg dominance were assessed using reliable measures of hand and foot preference $[23,24]$. Prior to formal chronometric testing, motor imagery vividness was assessed using the Kinesthetic and Visual Imagery Questionnaire, a reliable and valid assessment tool for persons with physical disability [17, 25]. This was followed by a chronometric test that compares the movement times during the imagination and execution of the stepping task [17, 18]. In this stepping task the subject is seated rather than standing [14] because stepping movements in sitting are easier for persons with severe motor and balance impairments. This stepping task in sitting involves hip flexion to lift the foot off the floor and knee extension-flexion to place the foot on the target and then to return it to the starting position. Thus, subjects sitting on a chair with a backrest with both feet resting on the floor were asked to imagine (I) and then execute physically (E) two series of five stepping movements. The movements consisted of placing one foot forward onto a board (target) and then returning it to the floor. The board $(41 \mathrm{~cm}$ wide $\times$ $26 \mathrm{~cm}$ long $\times 2 \mathrm{~cm}$ high) was placed transversely about $5 \mathrm{~cm}$ in front of the feet. The test-retest reliability of this chronometric test has been confirmed in 20 persons with stroke and 46 age-matched healthy controls [18]. During testing, subjects were required to close their eyes and to verbally signal each time they imagined touching the target. To promote motor imagery (internal perspective or firstperson perspective), they were instructed to see and feel moving their leg from the inside (i.e., as if they were really doing it and not watching from the outside like looking in a mirror) until the examiner told them to stop. Two series of five stepping movements were carried out with each leg. The imagination condition was presented first to minimize the possibility that the subject was influenced by the duration of the real movement or used a counting strategy. The same procedure was repeated for the second series. Each series and each condition (imagination and execution) were separated by a $30 \mathrm{~s}$ rest period. Prior to formal testing, there was a demonstration of the task and a practice run (physical and mental).

Three domains of working memory were assessed: visuospatial, verbal, and kinesthetic. We used a standardized procedure [26] that has been widely used with persons with brain injury [27]. The examiner presents a series of items and asks the subject to reproduce it immediately in the same order. For each domain, items are taken randomly from a 
limited pool of items and are presented sequentially. For each type of material, 5 lists of 2 items were first presented. If the subject could reproduce correctly 3 of the 5 lists, the list length was increased by 1 item otherwise, testing was interrupted. The verbal stimuli were taken from a set of 9 frequent and imaginable monosyllabic words presented in the auditory modality [28]. In the visuospatial condition, the examiner tapped on a series of 9 blocks (Corsi block-tapping task) presented in a random arrangement in front of the subjects. The subject was asked to reproduce the sequence by tapping on the same blocks $[29,30]$. In the kinesthetic condition, the same standardized procedure was used as previously but the stimuli were constructed to test working memory for movement [31,32]. The examiner produced passively a series of gestures, and the subject (blindfold) was asked to reproduce them. The gestures involved unilateral and bilateral lower-limb movements as well as movements involving the trunk and the upper and lower limbs.

2.3. Data Analysis. The mean duration of the two series of five movement repetitions was averaged for each limb and each condition. Imagination/execution (I/E) time ratios were calculated to quantify the temporal congruence between the two conditions. For working memory tests, the number of sequences and the number of items correctly recalled were converted to percent of maximal possible score and the percent score of the two parameters was averaged [31, 32]. To quantify the degree of temporal coupling (I/E) time ratios were computed $[17,18]$.

The age among groups was compared with the paired Student's $t$-test. Because the time after stroke was longer in the RHL subgroup than the LHL subgroup, an analysis of covariance taking into account the time after stroke was carried out with 2 within-subjects factors (limb side and condition) and one between-subjects factors (side of hemispheric lesion). Since there was no significant time interaction, further analyses using analysis of variance (ANOVA) for repeated measures were carried out. For each group (CTL and CVA), an ANOVA for repeated measures for two within-subjects factors: limb side (affected and unaffected) and condition (physical execution and mental simulation), followed by the post hoc Bonferroni procedure was carried out to examine whether temporal congruence was preserved. Then, to determine the effect of the side of the hemispheric lesion on temporal congruence, an ANOVA with repeated measures for two within-subjects factors: limb side (affected and unaffected) and condition (physical execution and mental simulation), and one between-subjects factors (left and right hemispheric lesion) followed by the post hoc Bonferroni procedure were conducted. To establish whether the degree of temporal congruence was comparable after an RHL and LHL, a similar analysis was carried out on the $\mathrm{I} / \mathrm{E}$ time ratios. Impairment of working memory ability was examined for each dimension between groups (CTL, RHL, LHL) with a one-way ANOVA. The relationship between imagination and execution times was studied with the $r$ Pearson product-moment correlation. The statistical level of significance was set at 0.05 . Statistical tests were performed with SPSS 11.0 for Windows.

\section{Results}

Subject characteristics are reported in Table 1. When available in the medical chart, results from four clinical tests (Timed-Up and Go, gait speed, Fugl-Meyer Sensorimotor Assessment, Balance Scale) are provided to give additional information about motor disability. There were no significant differences between groups for all variables except for the time after stroke which was significantly longer $(P=$ .01 ) in the subgroup of patients with an RHL; however, both groups were in a chronic stage ( 1 and 2 years after stroke). The visual motor imagery subscores were greater than corresponding kinesthetic imagery scores in all groups $(P=.004)$ except in the RHL subgroup that did not show the usual visual motor imagery dominance. The cerebrovascular accidents (CVAs) induced unilateral lesions (cortical and/or subcortical) that were confirmed by CT or MRI scans. The lesions were located in the left $(n=18)$ or the right $(n=19)$ hemisphere resulting in paresis of the contralateral side of the body (Table 2). All patients had right-hand dominance, and all but one patient in each group (Table 2: subject $18 \mathrm{R}$ and subject $4 \mathrm{~L}$ ) had right-foot dominance.

Results from the ANOVAs carried out for each group separately (Figure 1(a), left panel) showed that, in the CTL group, there was no significant difference in the movement times between limb side $\left(F_{(1,31)}=.015 ; P=.903\right)$ and condition $\left(F_{(1,31)}=2.249 ; P=.144\right)$ and no interaction $\left(F_{(1,31)}=.077 ; P=.783\right)$ between limb side and condition indicating a temporal coupling between imagination and execution conditions for both limbs. The temporal congruence between conditions is reflected by $\mathrm{I} / \mathrm{E}$ time ratios near 1 in the dominant and nondominant limb sides, respectively (mean: 1.09 and 1.10) (Figure 1(a), right panel). In contrast, in the CVA group (RHL and LHL: $n=37$ ) movement times were longer on the affected side than on the unaffected limb side (limb side effect: $F_{(1,36)}=10.6 ; P=.002$ ), and a significant effect of condition was also observed $\left(F_{(1,36)}=\right.$ 22.3; $P=.0001)$ with longer movement times during the imagined condition than the execution condition, but there was no limb side $x$ condition interaction $\left(F_{(1,36)}=.660\right.$; $P=.44$ ) (Figure 1(b), left panel). These findings imply that movements are slower on the affected limb side and that for both limbs they are slower during the imagination condition compared to the execution condition indicating a temporal uncoupling between imagination and execution conditions on both limb sides. The degree of temporal uncoupling is reflected in high $\mathrm{I} / \mathrm{E}$ time ratios on both the affected (mean: 1.23 and 1.34) and unaffected limbs, respectively, indicating that subjects overestimated by $23 \%$ and $34 \%$ the duration of stepping during the imagination condition (Figure 1(b) right panel).

To determine whether temporal uncoupling was linked to the side of the hemispheric lesion, further analyses comparing the subgroups of patients were carried out. The mean (SD) movement durations are illustrated for each limb side and condition in Figure 2(a). Results from the ANOVAs revealed a significant effect of limb side $\left(F_{(1,35)}=10.36 ; P=\right.$ $.003)$ and condition $\left(F_{(1,35)}=23.94 ; P=.0001\right)$ as well as a group $x$ condition interaction $\left(F_{(1,35)}=4.67 ; P=.037\right)$. 
TABLE 1: Subject characteristics.

\begin{tabular}{|c|c|c|c|c|}
\hline & CTL $(n=32)$ & CVA $(n=37)$ & RHL $(n=19)$ & LHL $(n=18)$ \\
\hline \multicolumn{5}{|l|}{ Age $(y)$} \\
\hline Mean & 59.0 & 60.1 & 61.5 & 58.5 \\
\hline SD & 10.6 & 8.0 & 8.8 & 7.0 \\
\hline Range & $37.6-77.6$ & $47.2-75.0$ & $48.1-75.0$ & $47.2-72.1$ \\
\hline \multicolumn{5}{|l|}{ Gender } \\
\hline Men & 14 & 27 & 15 & 12 \\
\hline Women & 18 & 10 & 4 & 6 \\
\hline \multicolumn{5}{|l|}{ Time since onset (mo) } \\
\hline Mean & NA & 24.0 & $34.4^{*}$ & 13.0 \\
\hline SD & & 26.8 & 32.1 & 13.2 \\
\hline Range & & $1.8-123.8$ & $1.8-123.8$ & $2.1-44.0$ \\
\hline \multicolumn{5}{|l|}{ KVIQ: visual (50) } \\
\hline Mean & $36.9^{* *}$ & $38^{* *}$ & 37.3 & $39.4^{* *}$ \\
\hline SD & 8 & 7.8 & 7.9 & 7.8 \\
\hline Range & $15-49$ & $16-50$ & $16-50$ & $25-50$ \\
\hline \multicolumn{5}{|l|}{ KVIQ: kinesthetic (50) } \\
\hline Mean & 32.2 & 34 & 34.8 & 34.1 \\
\hline SD & 8.6 & 9.7 & 10 & 9 \\
\hline Range & $15-48$ & $12-50$ & $12-49$ & $17-50$ \\
\hline Gait speed $(\mathrm{cm} / \mathrm{s})$ & & $(n=36)$ & $(n=18)$ & $(n=18)$ \\
\hline Mean & NA & 85.2 & 77.9 & 92.4 \\
\hline $\mathrm{SD}$ & & 36.4 & 35.0 & 37.2 \\
\hline Range & & $9.7-162$ & $9.7-133.7$ & $33.5-162$ \\
\hline Timed up and go (s) & & $(n=29)$ & $(n=14)$ & $(n=15)$ \\
\hline Mean & NA & 20.4 & 25.0 & 16.1 \\
\hline SD & & 15.2 & 19.7 & 7.8 \\
\hline Range & & $7.3-83$ & $8.4-83$ & $7.3-34.0$ \\
\hline Balance scale (56) & & $(n=35)$ & $(n=18)$ & $(n=17)$ \\
\hline Mean & NA & 49.7 & 47.7 & 51.8 \\
\hline SD & & 8.4 & 9.6 & 6.6 \\
\hline Range & & $23-56$ & $23-56$ & $31-56$ \\
\hline Fugl-Meyer (34) & & $(n=28)$ & $(n=14)$ & $(n=14)$ \\
\hline Mean & NA & 26.7 & 28.1 & 25.3 \\
\hline SD & & 6.5 & 5.8 & 7.0 \\
\hline Range & & $11-34$ & $16-34$ & $11-33$ \\
\hline
\end{tabular}

${ }^{*}$ Longer $(P=.01)$ time after stroke in the RHL group; ${ }^{* *}$ higher visual than kinesthetic scores within group $(P=.004)$; KVIQ: Kinesthetic and Visual Imagery Questionnaire; Fugl-Meyer Sensorimotor Assessment: motor subscore of the lower limb, Max value in brackets; NA: not applicable.

Post hoc analyses indicated that, in contrast to the LHL subgroup, in the RHL subgroup the movement times on both the affected and unaffected limb sides were longer during imagination $(P=.0001)$ than during execution, reflecting a bilateral temporal uncoupling. The degree of temporal uncoupling as revealed by I/E time ratios are illustrated in Figure 2(b). Results from the ANOVAs revealed a significant group $\left(F_{(1,35)}=6.99 ; P=.01\right)$ and limb side effect $\left(F_{(1,35)}=\right.$ 4.04; $P=.05)$ but no interaction $\left(F_{(1,35)}=.108 ; P=\right.$ .744). The findings also indicate that $\mathrm{I} / \mathrm{E}$ time ratios in the RHL subgroup on both the affected limb $(1.37 ; P=.01)$ and the unaffected limb $(1.50 ; P=.01)$ were higher than corresponding I/E time ratios in the LHL subgroup (1.09 and 1.12). Thus, subjects with an RHL were overestimated by $37 \%$ and $50 \%$ the duration of stepping movements during the imagination condition, compared to $9 \%$ and $12 \%$ in the LHL group. Figure 3 provides individual I/E time ratios for each subgroup arranged in an ascending order (subject numbers do not match those in Table 2) and the graphs illustrate the variability between patients. In each subgroup, some take more time to imagine than to execute stepping (ratio above 1 = overestimation) others take less time (ratio below 1 = underestimation). Note that while in the LHL subgroup, I/E time ratios on affected and unaffected limb 
TABLE 2: Lesion location.

\begin{tabular}{|c|c|c|c|c|c|c|c|}
\hline \multicolumn{4}{|c|}{ Right hemispheric lesion $(n=19)$} & \multicolumn{4}{|c|}{ Left hemispheric lesion $(n=18)$} \\
\hline \multirow{2}{*}{$N$} & \multirow{2}{*}{ Lesion location } & \multicolumn{2}{|c|}{$\mathrm{I} / \mathrm{E}$ time ratio } & \multirow{2}{*}{$N$} & \multirow{2}{*}{ Lesion location } & \multicolumn{2}{|c|}{$\mathrm{I} / \mathrm{E}$ time ratio } \\
\hline & & Aff. & Unaff. & & & Aff. & Unaff. \\
\hline $1 \mathrm{R}$ & MCA territory & 0.82 & 1.79 & $1 \mathrm{~L}$ & MCA territory & 0.69 & 0.85 \\
\hline $2 \mathrm{R}$ & Frontoparietal cortex & 0.95 & 0.79 & $2 \mathrm{~L}$ & MCA territory & 0.72 & 0.94 \\
\hline $3 \mathrm{R}$ & $\begin{array}{c}\text { Internal capsule, basal ganglia, and } \\
\text { corona radiate }\end{array}$ & 0.96 & 1.55 & $3 \mathrm{~L}$ & Internal capsule (post. limb) & 0.79 & 1.04 \\
\hline $4 \mathrm{R}$ & Frontal (post.) and temporal cortex & 0.96 & 1.19 & $4 \mathrm{~L}$ & $\begin{array}{l}\text { Frontal cortex (post.) and } \\
\text { subcortex }\end{array}$ & 0.83 & 1.03 \\
\hline $5 \mathrm{R}$ & MCA territory & 1.08 & 1.36 & $5 \mathrm{~L}$ & MCA territory & 0.85 & 0.90 \\
\hline $6 \mathrm{R}$ & External capsule and basal ganglia & 1.10 & 1.05 & $6 \mathrm{~L}$ & MCA territory & 0.98 & 0.89 \\
\hline $7 \mathrm{R}$ & MCA territory and thalamus & 1.12 & 1.05 & $7 \mathrm{~L}$ & $\begin{array}{c}\text { Frontoparietal cortex and basal } \\
\text { ganglia }\end{array}$ & 1.00 & 0.96 \\
\hline $8 \mathrm{R}$ & MCA territory & 1.14 & 1.21 & $8 \mathrm{~L}$ & Frontal cortex (post.) & 1.03 & 1.14 \\
\hline $9 \mathrm{R}$ & Frontoparietal (subcortical) & 1.24 & 1.27 & $9 \mathrm{~L}$ & Thalamocapsular & 1.10 & 1.20 \\
\hline $10 \mathrm{R}$ & MCA territory & 1.26 & 1.38 & $10 \mathrm{~L}$ & Parietotemporal cortex & 1.27 & 1.32 \\
\hline $11 \mathrm{R}$ & MCA territory & 1.30 & 1.54 & $11 \mathrm{~L}$ & Parietal cortex & 1.28 & 1.15 \\
\hline $12 \mathrm{R}$ & Internal capsule and corona radiate & 1.31 & 1.33 & $12 \mathrm{~L}$ & Frontal cortex and subcortex & 1.52 & 1.34 \\
\hline $13 \mathrm{R}$ & Temporolenticular & 1.46 & 1.63 & $13 \mathrm{~L}$ & $\begin{array}{l}\text { Frontotemporal cortex and } \\
\text { putamen }\end{array}$ & 1.53 & 1.64 \\
\hline $14 \mathrm{R}$ & MCA territory & 1.49 & 1.48 & $14 \mathrm{~L}$ & $\begin{array}{c}\text { Frontal cortex (post.), caudate } \\
\text { nucleus }\end{array}$ & 1.10 & 1.31 \\
\hline $15 \mathrm{R}$ & Frontoparietal cortex & 1.58 & 0.98 & $15 \mathrm{~L}$ & $\begin{array}{c}\text { Paraventricular from claustrum } \\
\text { to external capsule }\end{array}$ & 1.40 & 1.55 \\
\hline $16 \mathrm{R}$ & MCA territory & 1.54 & 2.67 & $16 \mathrm{~L}$ & $\begin{array}{l}\text { Frontal cortex and } \\
\text { intraventricular }\end{array}$ & 1.22 & 1.31 \\
\hline $17 \mathrm{R}$ & MCA territory & 1.76 & 1.76 & $17 \mathrm{~L}$ & Subcortex paraventricular & 1.07 & 1.11 \\
\hline $18 \mathrm{R}$ & MCA territory & 2.27 & 2.68 & $18 \mathrm{~L}$ & Temporooccipital cortex & 1.19 & 1.48 \\
\hline $19 \mathrm{R}$ & MCA territory & 2.53 & 1.70 & & & & \\
\hline
\end{tabular}

$N$ : patient number; I/E time ratio: imagination/execution time ratio; MCA: middle cerebral artery; aff.: affected limb; unaff.: unaffected limb.

sides were generally similar and were close to 1 , those in the RHL subgroup tended to differ more between limb sides with several values above 1.5. The latter difference between limb sides is reflected in the correlation coefficients of $r=.86$ and $r=.56$ computed between I/E ratios of the affected and unaffected limbs in the LHL and RHL subgroups, respectively.

Figure 4 illustrates the mean (1SD) visuospatial, kinesthetic, and verbal working memory performance for the subgroups of patients with a left (LHL) and a right (RHL) hemispheric lesion and for the control subjects (CTL). Results from the ANOVA indicate a significant decrease in performance for the visuospatial domain after an RHL $(P=$ .006) and a significant decrease in the kinesthetic domain after an LHL $(P=.006)$, while both subgroups had a significant deficit in the verbal domain (LHL: $P=.000$ and RHL: $P=.002)$. A near significant $(P=.06)$ correlation was found between visual working memory and the $\mathrm{I} / \mathrm{E}$ time ratio for the unaffected leg $(r=.25)$ for the whole group $(n=37)$; when individually examined, however, the correlation was stronger $(r=.35)$ in the RHL subgroup than the LHL subgroup $(r=.20)$ but did not reach statistical significance.

\section{Discussion}

The results indicate that patients with an RHL demonstrated temporal incongruence between real and imagined stepping movements. In contrast to CTL individuals and patients with an LHL, in the RHL group the imagination times during the imagination of stepping movements on the affected and unaffected side were, respectively, $37 \%$ and $50 \%$ longer than execution times (overestimation) indicating a bilateral slowing of motor imagery. Our findings concur with previous findings $[15,16]$ describing similar temporal incongruence for upper limb movements in a smaller sample of persons with RHL and thus extend the notion of a temporal deficit of motor imagery to movements of the lower limbs.

With the computation of $\mathrm{I} / \mathrm{E}$ time ratios it is possible to measure the level and direction (over- or underestimation of imagined movement times) of temporal incongruence which varies between subjects within each group. It is of note that more patients in the RHL group had high ratios above 1 (Figure 3 and Table 2). As shown in Table 2, the cerebral lesions were quite extensive and involved similar regions in 


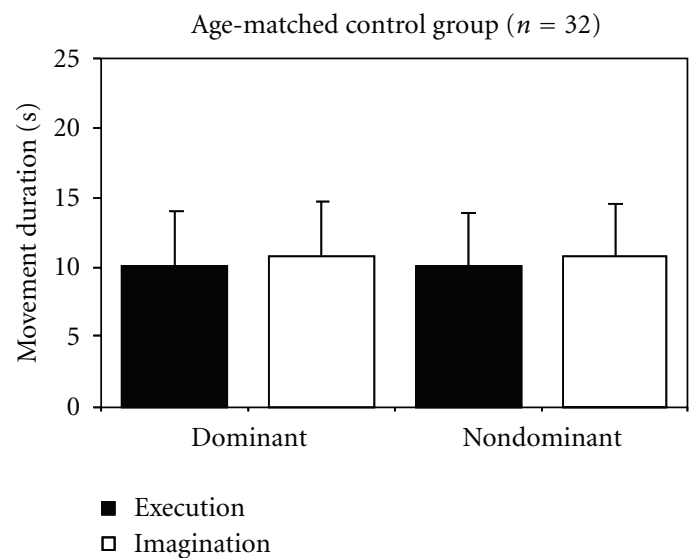

(a)

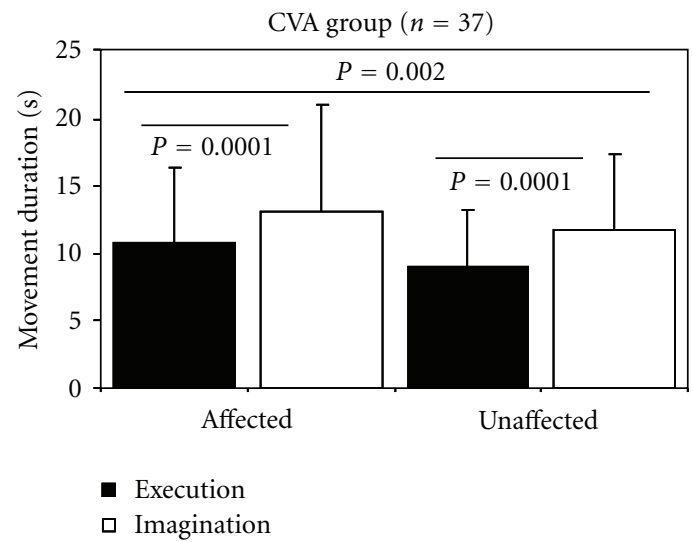

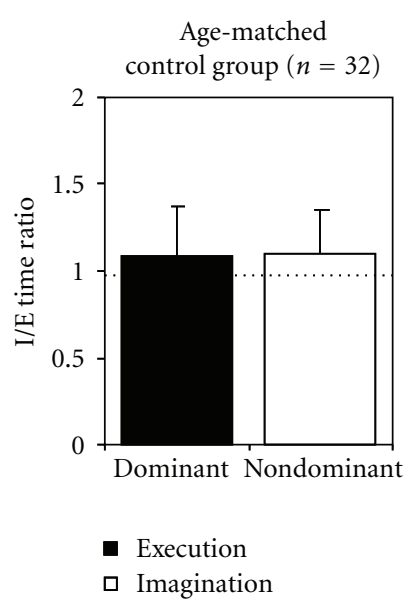

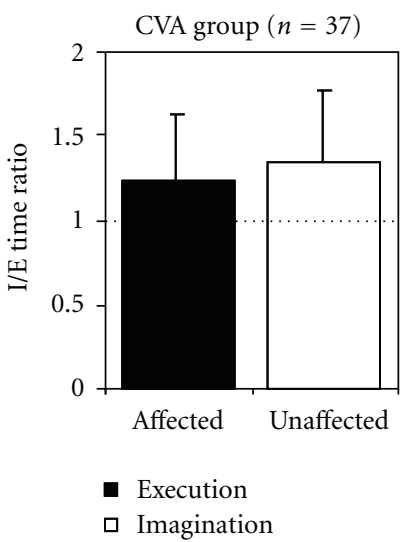

(b)

Figure 1: (a) Mean duration (1SD) of stepping movements during physical execution and mental simulation conditions (left panel) and imagination/execution time ratio of dominant and nondominant sides in age-matched healthy individuals. Similar duration during both conditions (temporal equivalence) yielding I/E time ratio near 1. (b) Similar illustration as in (a) for the stroke group. Longer stepping movement times during the imagination compared to the execution condition in individuals with stroke (total group); I/E time ratios above 1 indicating a lack of temporal congruence. Dotted lines indicate temporal congruence of 1 to 1 .

both groups, thus making it difficult to associate temporal incongruence with specific regions. However, note that I/E time ratios were highest in RHL patients with lesions located in the middle cerebral artery (MCA) territory. Conversely, MCA territory lesions in the LHL group yielded low I/E time ratios suggesting that lesions of the MCA territory in the right hemisphere damage areas critical to processes underlying motor imagery.

For instance, the extensive lesions may have damaged regions of the RH involved in time-keeping mechanisms. Indeed, Harrington et al. [33] found that durationperception deficits (overestimation) after RHL were associated with lesions in the premotor and prefrontal cortex known to be critical for working memory (Brodmann areas $6,8,9$, and 46), and lesions in the inferior parietal cortex essential for movement representation. They also found that, despite the similarity between the RHL and LHL subgroups in lesion loci and size, only RHLs were associated with a disruption in time discriminations [33].
Their results implicate a right hemisphere prefrontal-inferior parietal network in timing and suggest that time-dependent attention and working memory functions may contribute to temporal perception deficits observed after damage to this network [33, 34]. Likewise, the impairment of visuospatial working memory to a greater extent in the RHL could also have resulted from additional damage to the $\mathrm{RH}$ which is important for maintaining spatial information over time during motor imagery [21,22]. Although the visuospatial working memory deficit was greater in the RHL than the LHL subgroup, we could not establish a strong correlation between the level of temporal incongruence as measured by I/E time ratios and working memory deficit. Thus, further studies with a larger sample or a sample with a greater range of deficits would be needed to confirm the link between these factors.

Other examples of motor imagery deficits associated with RHL come from studies in children with cerebral palsy (CP). The study comparing motor imagery accuracy (hand 


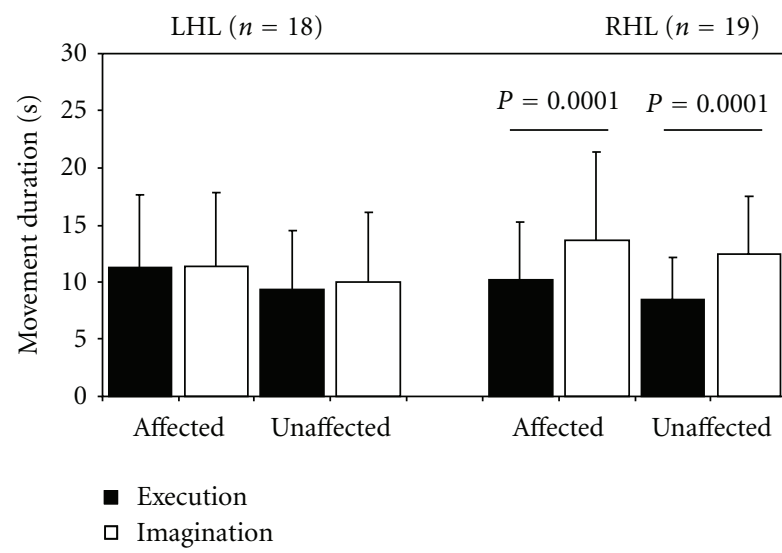

(a)

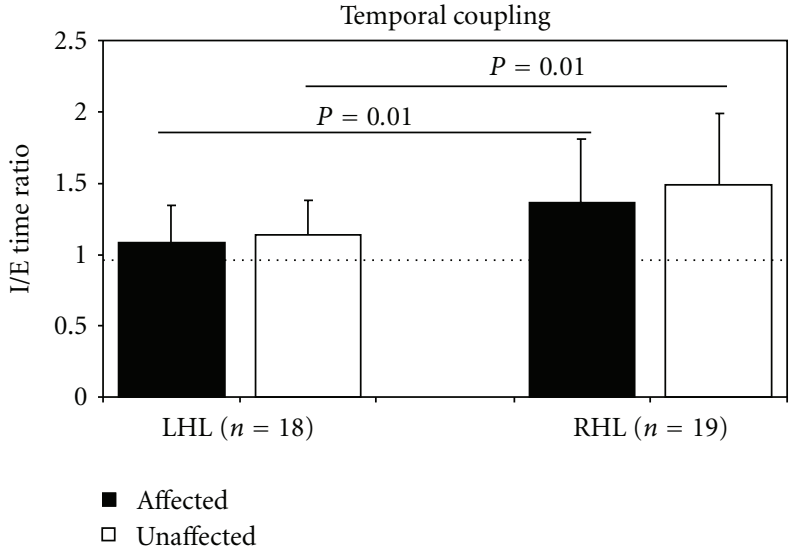

(b)

Figure 2: (a) Mean duration (1SD) of stepping movements during physical execution and mental simulation conditions on affected and unaffected sides in the subgroups of patients with a left hemispheric (LHL) and a right hemispheric (RHL) lesion. Note that the RHL subgroup, in contrast to the LHL subgroup, had longer movement times during the imagination condition. (b) Corresponding imagination/execution time ratios for dominant and nondominant sides in each subgroup of patients with an LHL or an RHL. Dotted line indicates temporal congruence of 1 to 1 .

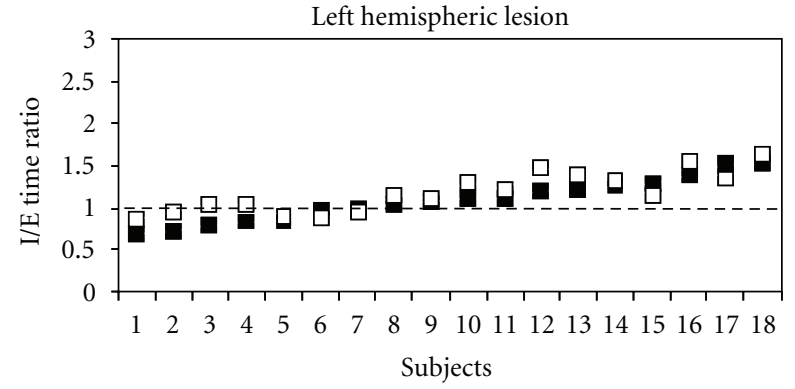

- Affected

$\square$ Unaffected

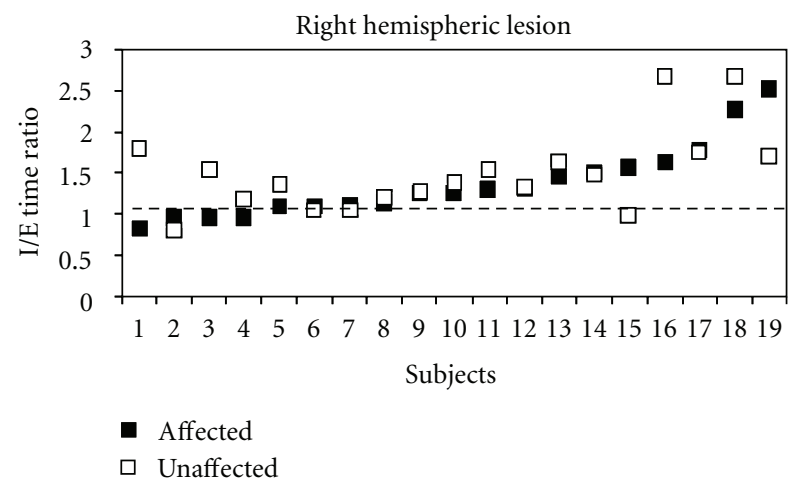

FIgURE 3: Illustration of individual I/E time ratio for the affected and unaffected sides in each subgroup of patients.

rotation task) in children with congenital hemiplegia [35] showed that children with an RHL were a little slower and less accurate in a hand rotation task compared to those with an LHL and that this deficit was also correlated with the functional level as measured by the Vineland Adaptive Behavior Scales. Lastly, findings from a recent case study of a

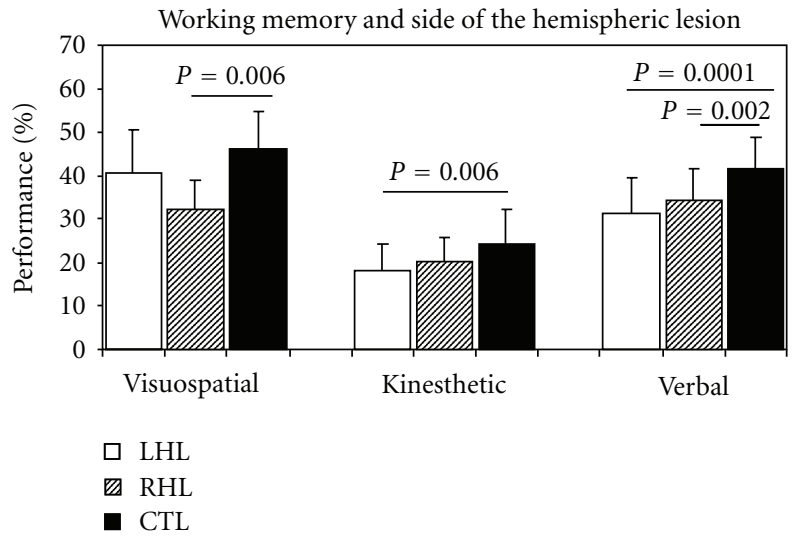

FIGURE 4: Mean (1SD) visuospatial, kinesthetic and verbal working memory performance for the sub-groups of patients with a left (LHL) and a right (RHL) hemispheric lesion and for the control subjects (CTL).

young boy with $\mathrm{CP}$ affecting the left side of his body provide evidence that children with $\mathrm{CP}$ have deficits in tasks involving visuospatial working memory and imagery ability [36] supporting the notion that visuospatial working memory is interconnected with the generation and maintenance of mental images [37-39].

The question arises as to the clinical significance of the slowing of motor imagery after RHL. It does not necessarily imply that patients with an RHL are unable to engage in motor imagery, but it does put into question the interpretation of mental chronometry outcomes. In fact, depending on the motor task or the context of mental practice, the duration of simulated movements can be overestimated (longer duration) or underestimated (shorter duration) even in normal subjects [9]. This means that it is essential to have 
comparative data from healthy subjects (especially for complex tasks) to control for these normal temporal variations. In the present study, normal subjects demonstrated temporal congruence for stepping movements, concurring with earlier findings for simple or highly automatic movements such as walking and grasping [9]. However, imagination times have been shown to increase up to $30 \%$ with task difficulty in healthy individuals $[2,9,10]$. Based on these observations, the overestimation of duration during imagined stepping after an RHL could indicate that the simulation of a simple motor task becomes more demanding after an RHL. In fact, although both subgroups had a good level of motor imagery vividness, the RHL group did not show the usual visual motor imagery dominance likely indicating that the generation of vivid images of the task was not as successful.

Mental chronometry is a simple tool that should be used on a regular basis to monitor the capacity of patients to reproduce mental tasks during motor imagery practice, especially for complex tasks with several sequences of movements such as activities of daily living (i.e., reaching for a glass and drinking, use of utensils, combing hair, eating, turning pages). This is important because in a recent study it was found that the imagination times could be 2 to 3 times shorter than execution times suggesting that the patients were not successfully engaged in mental rehearsal or did not understand the instructions [40]. Such underestimation of simulated movements may indicate some difficulty in representing mentally the task accurately as this has been documented in less experienced athletes for more complex tasks (i.e., skydiving and springboard diving), or when they rehearsed only one phase of complex movements [41, 42]. Thus, temporal incongruence should alert us as to whether a person has problems in movement representation or to comply with the instructions. It is also important to take into account the factors (i.e., task, context, experience) that normally influence temporal congruence. Lastly, based on present results severe temporal incongruence may indicate that the use of mental practice through motor imagery will have limitations in patients with a right hemisphere stroke.

In comparison to the control group, both subgroups of patients showed a decline in verbal working memory which should not be surprising since imaging studies $[43,44]$ have demonstrated that bilateral parietal regions are engaged when verbal information has to be recalled from shortterm memory [45]. On the other hand, the greater working memory deficit in the kinesthetic domain found after LHL is in keeping with the role of the left hemisphere in short-term maintenance of kinesthetic information [46].

Present findings are limited to adults with subacute and chronic ischemic strokes not involving the cerebellum or midbrain and without severe complications such as aphasia, neglect, or apraxia. Moreover, the findings cannot be extrapolated to more complex motor tasks or tasks involving the upper extremities. Also, there was one control subject ten years younger than the youngest patient. However, because the mean and median values were very close in both groups, it should not have too great an impact on the outcomes, especially since the main findings were based on comparisons made between subgroups of patients with similar age range, mean and median values. Another potential limitation is that the two groups of patients were not matched for stroke location and that the lesions were not focalized as in the Harrington et al. study [33]. Lastly, the time since stroke in the RHL subgroup was longer than in the LHL; however, statistical analysis with time after stroke as a covariable did not reveal a significant effect on movement duration and thus time after lesion should not have affected present findings, especially since both groups were in a chronic stage (mean: 12 and mean: 24 months).

\section{Conclusion}

Although motor imagery vividness is preserved after RHL, patients take more time to imagine than to execute physically stepping movements, indicating that the temporal congruence between real and imagined movement is not maintained. In addition, visuospatial working memory deficits were greater after an RHL than an LHL. Based on the analysis of lesion location and corresponding level of temporal incongruence, it is likely that extensive lesions in the RHL have damaged areas critical to processes underlying motor imagery. Mental chronometry is a simple method easy to use on a regular basis to monitor the capacity of patients to reproduce mentally motor tasks during mental practice. Temporal differences between real and imagined movement times for simple stepping movements, as evidenced here after an RHL, underline the need to use mental chronometry to control for possible temporal aberrations, especially during more complex tasks $[41,42]$.

\section{Acknowledgments}

The authors thank the subjects who participated in this study. This work was supported by Quebec Provincial Rehabilitation Research Network (FRSQ) and the CIHR.

\section{References}

[1] J. Decety and J. Grèzes, "Neural mechanisms subserving the perception of human actions," Trends in Cognitive Sciences, vol. 3, no. 5, pp. 172-178, 1999.

[2] J. Decety, M. Jeannerod, and C. Prablanc, "The timing of mentally represented actions," Behavioural Brain Research, vol. 34, no. 1-2, pp. 35-42, 1989.

[3] J. Decety and M. Jeannerod, "Mentally simulated movements in virtual reality: does Fitt's law hold in motor imagery?" Behavioural Brain Research, vol. 72, no. 1-2, pp. 127-134, 1996.

[4] J. Decety, M. Jeannerod, M. Germain, and J. Pastene, "Vegetative response during imagined movement is proportional to mental effort," Behavioural Brain Research, vol. 42, no. 1, pp. 1-5, 1991.

[5] S. Fusi, D. Cutuli, M. R. Valente, P. Bergonzi, C. A. Porro, and P. E. Di Prampero, "Cardioventilatory responses during real or imagined walking at low speed," Archives Italiennes de Biologie, vol. 143, no. 3-4, pp. 223-228, 2005.

[6] M. F. Lafleur, P. L. Jackson, F. Malouin, C. L. Richards, A. C. Evans, and J. Doyon, "Motor learning produces parallel dynamic functional changes during the execution and imagination of sequential foot movements," NeuroImage, vol. 16, no. 1, pp. 142-157, 2002. 
[7] F. Malouin, C. L. Richards, P. L. Jackson, F. Dumas, and J. Doyon, "Brain activations during motor imagery of locomotor-related tasks: a PET study," Human Brain Mapping, vol. 19, no. 1, pp. 47-62, 2003.

[8] J. Munzert and K. Zentgraf, "Motor imagery and its implications for understanding the motor system," Progress in Brain Research, vol. 174, pp. 219-229, 2009.

[9] A. Guillot and C. Collet, "Duration of mentally simulated movement: a review," Journal of Motor Behavior, vol. 37, no. 1, pp. 10-20, 2005.

[10] M. Jeannerod, "Mental imagery in the motor context," Neuropsychologia, vol. 33, no. 11, pp. 1419-1432, 1995.

[11] J. Decety and D. Boisson, "Effect of brain and spinal cord injuries on motor imagery," European Archives of Psychiatry and Neurological Sciences, vol. 240, no. 1, pp. 39-43, 1990.

[12] A. Sirigu, L. Cohen, J. R. Duhamel et al., "Congruent unilateral impairments for real and imagined hand movements," NeuroReport, vol. 6, no. 7, pp. 997-1001, 1995.

[13] A. Sirigu, J. R. Duhamel, L. Cohen, B. Pillon, B. Dubois, and Y. Agid, "The mental representation of hand movements after parietal cortex damage," Science, vol. 273, no. 5281, pp. 1564 1566, 1996.

[14] F. Malouin, C. L. Richards, J. Desrosiers, and J. Doyon, "Bilateral slowing of mentally simulated actions after stroke," NeuroReport, vol. 15, no. 8, pp. 1349-1353, 2004.

[15] M. Sabaté, B. González, and M. Rodríguez, "Brain lateralization of motor imagery: motor planning asymmetry as a cause of movement lateralization," Neuropsychologia, vol. 42, no. 8 , pp. 1041-1049, 2004.

[16] C. M. Stinear, M. K. Fleming, P. A. Barber, and W. D. Byblow, "Lateralization of motor imagery following stroke," Clinical Neurophysiology, vol. 118, no. 8, pp. 1794-1801, 2007.

[17] F. Malouin, C. L. Richards, A. Durand, and J. Doyon, "Clinical assessment of motor imagery after stroke," Neurorehabilitation and Neural Repair, vol. 22, no. 4, pp. 330-340, 2008.

[18] F. Malouin, C. L. Richards, A. Durand, and J. Doyon, "Reliability of mental chronometry for assessing motor imagery ability after stroke," Archives of Physical Medicine and Rehabilitation, vol. 89, no. 2, pp. 311-319, 2008.

[19] F. Malouin and C. L. Richards, "Mental practice for relearning locomotor skills," Physical Therapy, vol. 90, no. 2, pp. 240-251, 2010.

[20] S. M. Kosslyn, V. Maljkovic, S. E. Hamilton, G. Horwitz, and W. L. Thompson, "Two types of image generation: evidence for left and right hemisphere processes," Neuropsychologia, vol. 33, no. 11, pp. 1485-1510, 1995.

[21] M. van Asselen, R. P. C. Kessels, S. F. W. Neggers, L. J. Kappelle, C. J. M. Frijns, and A. Postma, "Brain areas involved in spatial working memory," Neuropsychologia, vol. 44, no. 7, pp. 11851194, 2006.

[22] P. M. Corballis, "Visuospatial processing and the righthemisphere interpreter," Brain and Cognition, vol. 53, no. 2, pp. 171-176, 2003.

[23] R. C. Oldfield, "The assessment and analysis of handedness: the Edinburgh inventory," Neuropsychologia, vol. 9, no. 1, pp. 97-113, 1971.

[24] J. P. Chapman, L. J. Chapman, and J. J. Allen, "The measurement of foot preference," Neuropsychologia, vol. 25, no. 3, pp. 579-584, 1987.

[25] F. Malouin, C. L. Richards, P. L. Jackson, M. F. Lafleur, A. Durand, and J. Doyon, "The kinesthetic and visual imagery questionnaire (KVIQ) for assessing motor imagery in persons with physical disabilities: a reliability and construct validity study," Journal of Neurologic Physical Therapy, vol. 31, no. 1, pp. 20-29, 2007.

[26] O. Spreen and E. Strauss, A Compendium of Neuropsychological Tests, Oxford University Press, New York, NY, USA, 2nd edition, 1998.

[27] G. G. Vallar and T. Shallice, Neuropsychological Impairments of Shortterm Memory, Cambridge University Press, Cambridge, UK, 1990.

[28] J. Chatelois, H. Pineau, and S. Belleville, "Batterie informatisée d'évaluation de la mémoire inspirée de l'approche cognitive," Canadian Psychology, vol. 34, no. 1, pp. 45-63, 1993.

[29] E. De Renzi, P. Faglioni, and P. Previdi, "Spatial memory and hemispheric locus of lesion," Cortex, vol. 13, no. 4, pp. 424434, 1977.

[30] R. P. Kessels, M. J. van Zandvoort, A. Postma, L. J. Kappelle, and E. H. de Haan, "The corsi block-tapping task: standardization and normative data," Applied Neuropsychology, vol. 7, no. 4, pp. 252-258, 2000.

[31] F. Malouin, S. Belleville, J. Desrosiers, J. Doyon, and C. L. Richards, "Working memory and mental practice outcomes after stroke," Archives of Physical Medicine and Rehabilitation, vol. 85, no. 2, pp. 177-183, 2004.

[32] F. Malouin, C. L. Richards, and A. Durand, "Normal aging and motor imagery vividness: implications for mental practice training in rehabilitation," Archives of Physical Medicine and Rehabilitation, vol. 91, no. 7, pp. 1122-1127, 2010.

[33] D. L. Harrington, K. Y. Haaland, and R. T. Knight, "Cortical networks underlying mechanisms of time perception," Journal of Neuroscience, vol. 18, no. 3, pp. 1085-1095, 1998.

[34] D. L. Harrington, R. R. Lee, L. A. Boyd, S. Z. Rapcsak, and R. T. Knight, "Does the representation of time depend on the cerebellum? Effect of cerebellar stroke," Brain, vol. 127, no. 3, pp. 561-574, 2004.

[35] J. Williams, S. M. Reid, D. S. Reddihough, and V. Anderson, "Motor imagery ability in children with congenital hemiplegia: effect of lesion side and functional level," Research in Developmental Disabilities, vol. 32, no. 2, pp. 740-748, 2010.

[36] L. Barca, F. Frascarelli, G. Pezzulo et al., "Working memory and mentalimagery in Cerebral Palsy. A single case investigation," Neurocase. In press.

[37] A. D. Baddeley, Working Memory, Oxford University Press, New-York, NY, USA, 1986.

[38] C. Cornoldi, R. Dalla Vecchia, and P. E. Tressoldi, "Visualspatial working memory limitations in low visuo-spatial high verbal intelligence children," Journal of Child Psychology and Psychiatry and Allied Disciplines, vol. 36, no. 6, pp. 1053-1064, 1995.

[39] C. Cornoldi, F. Rigoni, P. E. Tressoldi, and C. Vio, "Imagery deficits in nonverbal learning disabilities," Journal of Learning Disabilities, vol. 32, no. 1, pp. 48-57, 1999.

[40] A. J. Wu, V. Hermann, J. Ying, and S. J. Page, "Chronometry of mentally versus physically practiced tasks in people with stroke," American Journal of Occupational Therapy, vol. 64, no. 6, pp. 929-934, 2010.

[41] C. Calmels, P. Holmes, E. Lopez, and V. Naman, "Chronometric comparison of actual and imaged complex movement patterns," Journal of Motor Behavior, vol. 38, no. 5, pp. 339348, 2006.

[42] C. L. Reed, "Chronometric comparisons of imagery to action: visualizing versus physically performing springboard dives," Memory and Cognition, vol. 30, no. 8, pp. 1169-1178, 2002.

[43] E. E. Smith and J. Jonides, "Neuroimaging analyses of human working memory," Proceedings of the National Academy of 
Sciences of the United States of America, vol. 95, no. 20, pp. 12061-12068, 1998.

[44] C. R. Clark, G. F. Egan, A. C. McFarlane et al., "Updating working memory for words: a PET activation study," Human Brain Mapping, vol. 9, no. 1, pp. 42-54, 2000.

[45] S. M. Ravizza, M. Behrmann, and J. A. Fiez, "Right parietal contributions to verbal working memory: spatial or executive?" Neuropsychologia, vol. 43, no. 14, pp. 2057-2067, 2005.

[46] K. Fiehler, M. Burke, A. Engel, S. Bien, and F. Rösler, "Kinesthetic working memory and action control within the dorsal stream," Cerebral Cortex, vol. 18, no. 2, pp. 243-253, 2008. 


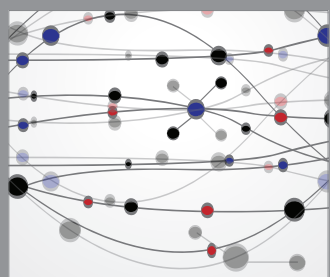

The Scientific World Journal
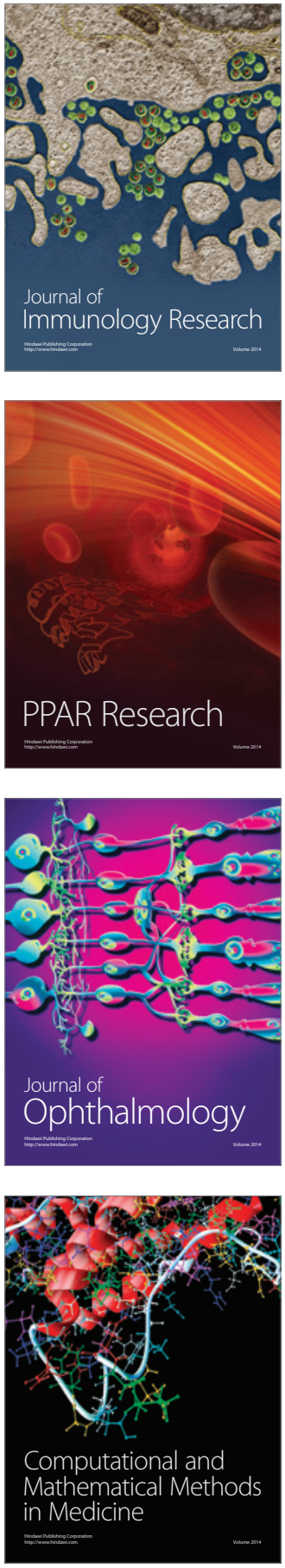

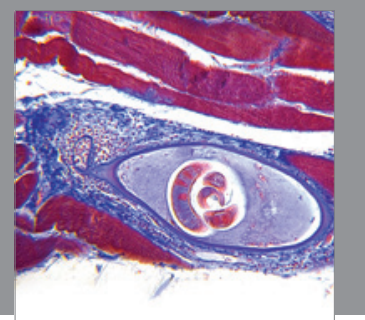

Gastroenterology

Research and Practice
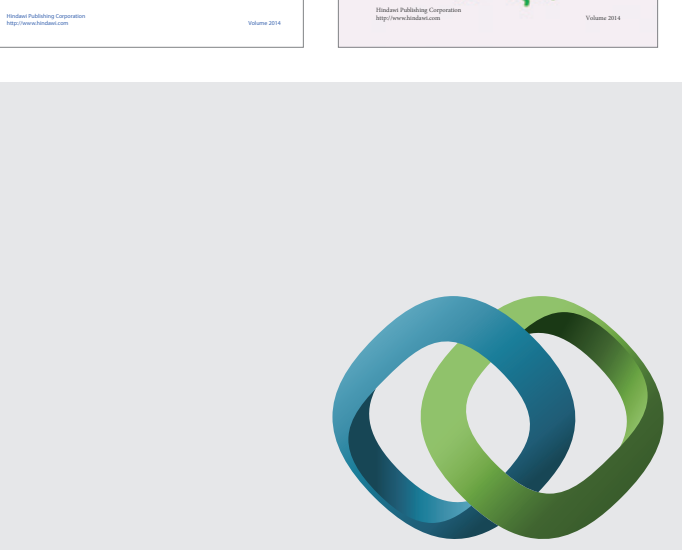

\section{Hindawi}

Submit your manuscripts at

http://www.hindawi.com
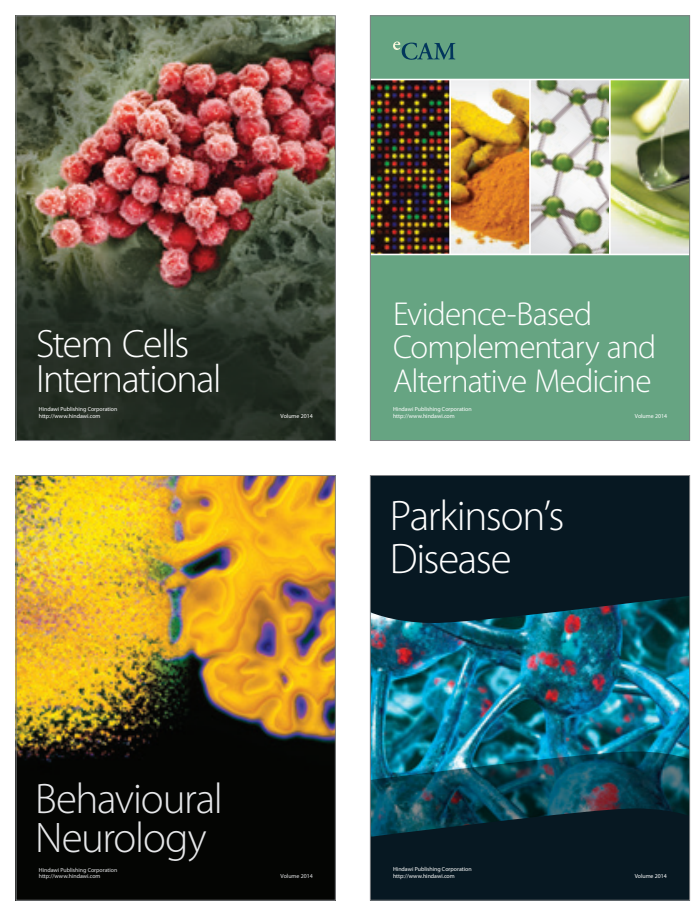

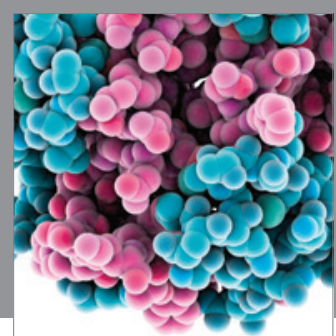

Journal of
Diabetes Research

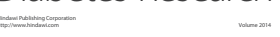

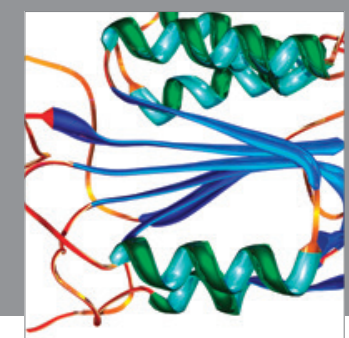

Disease Markers
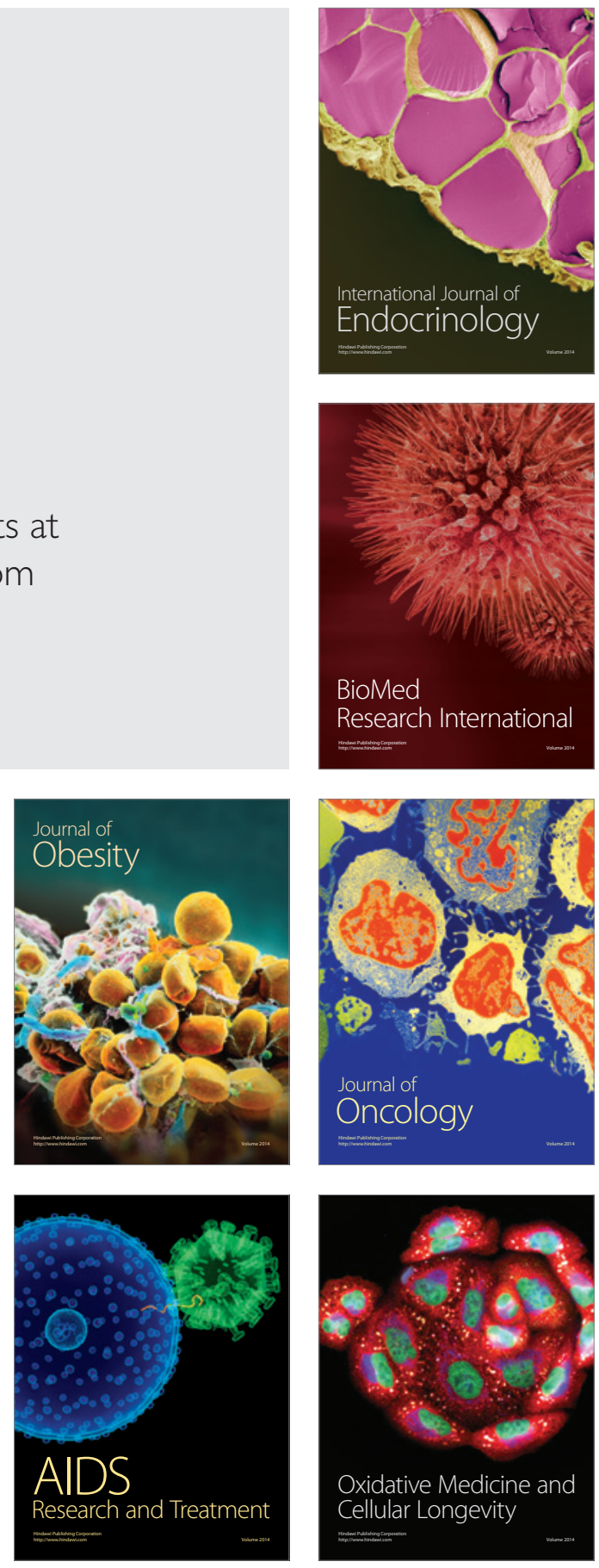